\title{
Abordagens clínicas associadas ao atendimento inicial do paciente politraumatizado: Revisão de literatura
}

Clinical approaches associated with the initial care of multiple trauma patients: Literature review Enfoques clínicos asociados con la atención inicial de pacientes politraumatizados: revisión de la

\section{literatura}

Recebido: 27/12/2020 | Revisado: 28/12/2020 | Aceito: 29/12/2020 | Publicado: 03/01/2021

Gabriela Alves dos Santos

ORCID: https://orcid.org/0000-0001-5939-0953

Universidade Tiradentes, Brasil

E-mail: gabriela.alvess1@ hotmail.com

Ana Luiza Medeiros Cesar

ORCID: https://orcid.org/0000-0003-2231-306X

Universidade Federal Fluminense, Brasil

E-mail: analumcesar@gmail.com

Felipe José Menezes Machado Santos

ORCID: https://orcid.org/0000-0002-0571-8893

Universidade Tiradentes, Brasil

E-mail: felipemachado_santos@hotmail.com

Fernando Matheus Santana Tunel

ORCID: https://orcid.org/0000-0001-7340-117X

Universidade Tiradentes, Brasil

E-mail: nandotunel@gmail.com

Gustavo Dultra Todt

ORCID: https://orcid.org/0000-0003-2208-8317

Universidade Tiradentes, Brasil

E-mail: gugatodt@hotmail.com

Igor José de Andrade Souza

ORCID: https://orcid.org/0000-0003-4407-5699

Universidade Tiradentes, Brasil

E-mail: igor.odonto.ufs@gmail.com

Ísis Suzanne Crisóstomo dos Santos ORCID: https://orcid.org/0000-0002-5991-0143

Universidade Tiradentes, Brasil

E-mail: isissuzanne_@hotmail.com

Joana Ferreira Rodrigues Santos ORCID: https://orcid.org/0000-0002-7945-7163 Universidade Tiradentes, Brasil E-mail: joanaf863@gmail.com

Julyani Mota Souza Loeser

ORCID: https://orcid.org/0000-0002-8748-9456 Universidade Tiradentes, Brasil

E-mail: julyaniloeser@ outlook.com

Laíse Oliveira Melo

ORCID: https://orcid.org/0000-0001-5321-1096 Universidade Tiradentes, Brasil

E-mail: 1aise.melo.98@gmail.com

Layna Gabriely Muniz Santos ORCID: https://orcid.org/0000-0002-0302-0950 Universidade Tiradentes, Brasil

E-mail: laynagabrielly@hotmail.com Louise Cristina Santos

ORCID: https://orcid.org/0000-0001-7458-975X Universidade Tiradentes, Brasil

E-mail: louise_cristina123@ hotmail.com

Mateus Ferreira Nogueira

ORCID: https://orcid.org/0000-0003-2417-1309 Universidade Tiradentes, Brasil

E-mail: mateusffnn@gmail.com

Maurício Santos Santana

ORCID: https://orcid.org/0000-0001-5340-4103 Universidade Tiradentes, Brasil

E-mail: mauriciosanttana10@gmail.com 


\author{
Vanessa Gonçalves Moreira \\ ORCID: https://orcid.org/0000-0002-9471-0649 \\ Universidade Tiradentes, Brasil \\ E-mail: vanessagoncaalves@outlook.com \\ Vitória Virgínia Maria Machado Vanderley \\ ORCID: https://orcid.org/0000-0001-8972-3595 \\ Universidade Tiradentes, Brasil \\ E-mail: vitoriavirginiamachado@gmail.com \\ William José e Silva Filho \\ ORCID: https://orcid.org/0000-0002-2117-3352 \\ Universidade Federal de Sergipe, Brasil \\ E-mail: williamjsfilho10@gmail.com
}

\begin{abstract}
Resumo
$\mathrm{O}$ atendimento inicial aos pacientes vítimas de traumas requer agilidade e habilidade por meio de uma equipe altamente capacitada, onde a abordagem seja efetiva, diminuindo os riscos de uma possível evolução ao óbito do paciente. O presente estudo teve como objetivo avaliar os conceitos atuais relacionados as abordagens clinicas associadas ao atendimento inicial do paciente politraumatizado. Foi realizada uma revisão literatura integrativa da literatura com o intuito de sintetizar os conhecimentos pertencentes ao tema. A pesquisa foi realizada nas bases de dados da Pubmed e Periódicos CAPES entre os anos de 2003 e 2019, tendo como descritores: "Cuidados de Suporte Avançado de Vida no Trauma", "Mortalidade" e "Traumatismo Múltiplo". Dezesseis produções científicas que atenderam aos critérios de inclusão e foram selecionados. A região da face, por apresentar uma anatomia proeminente, com grande exposição e pouca proteção, acaba sendo a área mais afetada, sendo responsável por 35 a $45 \%$ do acometimento em pacientes. O sistema ATLS (Advanced Trauma Life of Support) tem o intuito de esquematizar e padronizar a abordagem inicial ao paciente politraumatizado Dessa forma, é imprescindível um atendimento ágil e efetivo aos pacientes politraumatizados, para que o diagnóstico e tratamento do paciente seja bem-sucedido.
\end{abstract}

Palavras-chave: Cuidados de suporte avançado de vida no trauma; Mortalidade; Traumatismo múltiplo.

\begin{abstract}
Initial care for trauma victims requires agility and skill through a highly trained team, where the approach is effective, reducing the risks of a possible evolution to the patient's death. The present study aimed to evaluate current concepts related to clinical approaches associated with the initial care of polytrauma patients. An integrative literature review of the literature was carried out in order to synthesize the knowledge pertaining to the theme. The research was carried out in the databases of Pubmed and CAPES Periodicals between the years 2003 and 2019, having as descriptors: "Trauma", "mortality" and "polytrauma". Sixteen scientific productions that met the inclusion criteria and were selected. The region of the face, for presenting a prominent anatomy, with great exposure and little protection, ends up being the most affected area, being responsible for 35 to $45 \%$ of the involvement in patients. The ATLS (Advanced Trauma Life of Support) system aims to outline and standardize the initial approach to polytrauma patients. Therefore, an agile and effective care for polytrauma patients is essential for the diagnosis and treatment of the patient to be successful.
\end{abstract}

Keywords: Advanced trauma life support care; Mortality; Multiple trauma.

\title{
Resumen
}

La atención inicial a víctimas de trauma requiere agilidad y habilidad a través de un equipo altamente capacitado, donde el abordaje es efectivo, reduciendo los riesgos de una posible evolución a la muerte del paciente. El presente estudio tuvo como objetivo evaluar conceptos actuales relacionados con los abordajes clínicos asociados a la atención inicial de pacientes politraumatizados. Se realizó una revisión bibliográfica integradora de la literatura con el fin de sintetizar los conocimientos pertenecientes al tema. La investigación se realizó en las bases de datos de Pubmed Medicine y Revistas CAPES entre los años 2003 y 2019, teniendo como descriptores: "Trauma", "Mortalidad" y "politrauma". Dieciséis producciones científicas que cumplieron con los criterios de inclusión y fueron seleccionadas. La región del rostro, por presentar una anatomía prominente, con gran exposición y poca protección, acaba siendo la zona más afectada, siendo responsable del 35 al 45\% de la afectación. en pacientes. El sistema ATLS (Advanced Trauma Life of Support) tiene como objetivo perfilar y estandarizar el abordaje inicial de los pacientes politraumatizados, por lo que una atención ágil y eficaz a los pacientes politraumatizados es fundamental para el éxito del diagnóstico y tratamiento del paciente.

Palabras clave: Atención de apoyo vital avanzado en trauma; Mortalidad; Traumatismo múltiple.

\section{Introdução}

Nas últimas décadas, o trauma tem sido um quesito bastante considerável no panorama de saúde mundial. No Brasil, está no ranking entre as principais causas de mortalidade e altos índices de morbidade. Segundo dados da Organização Mundial de Saúde, a nível mundial, a cada dia morrem 16.000 pessoas em decorrência de trauma (Affonso et al., 2017). Em 
2018, a Organização Mundial de Saúde durante a "World Heatlh Statistics 2018” apresentou estatísticas e metas que visam até o ano de 2030, diminuir consideravelmente os índices de mortalidade, morbidade e fatores de risco à saúde. Está entre uma das metas, reduzir pela metade as lesões e mortes em todo o mundo, ocasionadas por acidentes de trânsito, onde em 2013, chegaram a 1,25 milhões de mortes (Mierzwa, Netto \& Oliveira, 2015).

Está elencado como principais situações causadoras do trauma: acidentes de trânsito (24,9\%), queda da própria altura $(24,7 \%)$ e agressões físicas $(18,4 \%)$. O paciente que se enquadra no termo politraumatizado apresenta lesões graves, por muitas vezes múltiplas, onde o seu estado de saúde está intimamente relacionado com a prontidão e eficácia da equipe de saúde que lhe dará suporte de vida, principalmente, durante o atendimento inicial. A vasta ocorrência de incidentes gerados pelo trauma afeta também a saúde pública, considerando a sua magnitude e relevância, onde um paciente politraumatizado irá demandar para o Estado, estrutura, assistência, suprimentos e tecnologias (Affonso et al., 2017; Galvão, Rodrigues \& Santana, 2017).

Visando uma uniformização e maior efetividade do atendimento de urgência dos hospitais, o Colégio Americano de Cirurgiões, com o intuito de padronizar o atendimento ao politraumatizado, criou o sistema ABCDE do Advanced Trauma Life Support (ATLS) (Galvão, Rodrigues \& Santana, 2017). Outro importante esquema bastante utilizado aos pacientes politraumatizados durante o seu atendimento inicial, é a Escala de Glasgow, que foi criada em 1974 no Instituto de Ciências Neurológicas de Glasgow, localizado na Inglaterra. Tal esquema é um artifício para avaliação de respostas neurológicas em um paciente traumatizado, frente aos estímulos que lhe são oferecidos (Oliveira, 2017). Dentre as inúmeras possibilidades do trauma acometer diversas regiões do corpo, o trauma de face, requer uma atenção especial. A região facial detém de uma grande área de exposição e pouca proteção, tornando-a mais susceptível aos traumas. Avaliou-se como lesões mais frequentes, as fraturas de mandíbula e lacerações faciais (Affonso et al., 2017).

Os traumas que ocorrem na região de face, por muitas vezes são uma combinação de estruturas que são acometidas durante a lesão, sendo utilizado um sistema de classificação que recebe a nomenclatura de I, II, III de Le Fort. Por acometerem estruturas nobres, necessitam de um atendimento emergencial com efetividade e agilidade. Diante as complicações desse tipo de trauma, duas merecem notoriedade: fraturas de base de crânio e obstrução de vias aéreas, já que as mesmas, envolvem estruturas primordiais para a preservação da saúde do paciente (Oliveira, 2017). Diante do exposto, o presente estudo teve como objetivo avaliar os conceitos atuais relacionados as abordagens clinicas associadas ao atendimento inicial do paciente politraumatizado.

\section{Metodologia}

O presente estudo foi realizado e fundamentado a partir de pesquisa bibliográfica, nas respectivas bases de dados: Pubmed e Periódicos CAPES entre os anos de 2003 e 2019. Os critérios de inclusão utilizados foram textos disponíveis online publicados na língua inglesa e portuguesa. Foi realizada a pesquisa nos DeCS (Descritores em Ciências da Saúde) para escolha dos descritores: "Cuidados de Suporte Avançado de Vida no Trauma", "Mortalidade" e "Traumatismo Múltiplo". Em seguida, os títulos foram selecionados pelo resumo e por relevância nos resultados da pesquisa, sendo excluídos do estudo os documentos indisponíveis para acesso na íntegra, dezesseis produções científicas que atenderam aos critérios de inclusão e foram selecionados. Posteriormente, mediante o reconhecimento, seleção e ordenação das informações dos documentos, realizou-se o processo de leitura do material viabilizando o entendimento e a compreensão em relação ao que o autor afirma com o problema para o qual se almeja resposta.

\section{Resultados}

Nas décadas antecedentes a de 70, não havia sistematização nas Unidades de Urgência e Emergência quanto ao atendimento aos pacientes politraumatizados. A equipe de saúde que atendia a tais vítimas não era capacitada, e nem obtinha 
de protocolos de atendimento. No ano de 1978, nos Estados Unidos, investiu-se em capacitação para médicos e enfermeiros, com a realização do primeiro curso de ATLS, a fim de tornar a equipe apta a prestar atendimentos de urgência e emergência em tempo hábil, e de forma efetiva (Guilherme et al., 2014).

Em meados da década de 80, foi criada a Sociedade Brasileira de Enfermeiros do Trauma (SOBET), capacitando os enfermeiros e dando enfoque ao atendimento de urgência e emergência (Guilherme et al., 2014). Entre 1990 e 1999, 79\% de todos os pacientes que apresentavam trauma como fratura dos ossos do corpo, em Washington (EUA), sofreram violência interpessoal sobre forma de agressão. Seguido pelos acidentes com veículos ato motor (6\%) e PAF (Ferimento por Arma de fogo) com outro 6\%, outras causas (esportes, quedas, e causas desconhecidas são responsável por 10\% (Casulari \& Flores, 2003).

Botter, Parreira \& Wulkan (2005) relata que os dois principais mecanismos de trauma são violência interpessoal e queda. Leis rigorosas de controle de velocidade, uso obrigatório de cinto de segurança e uso de air bag, quando disponível são creditados ao decréscimo do número de fratura associado ao trânsito. Enfatiza-se então, o quanto é inquestionável a necessidade de uma equipe de saúde para atendimento emergencial completa, capacitada e apta para atendimentos aos pacientes vítimas de politraumatismo. Somando-se a equipe, faz-se necessário a implementação de protocolos de atendimento, que viabilizem condutas a fim de estabelecer inicialmente o equilíbrio fisiológico da vítima, através da priorização da gravidade (Casulari \& Flores, 2003).

A partir de uma cronologia lógica de condutas e protocolos de prevenção, tratamentos devem ser estabelecidos, para que a identificação de lesões ou possíveis evoluções das mesmas possam ser evitadas, desde o atendimento primário, possibilitando ao paciente, redução no índice de sequelas ou possíveis óbitos, preconizando um estado de saúde estável (Campos, 2015). O paciente que necessita de atendimentos médicos de caráter de urgência e emergência, normalmente encontra-se em situações que se faz fundamental a assistência médica de alta e média complexidade, além de estar em um estado onde tal atendimento acarreta o acontecimento de riscos, que podem ser gerados ao paciente uma evolução para sérios eventos adversos, envolvendo perigo ao paciente. Cerca de 70\% desses eventos, concomitantemente com os erros constatados, geralmente em ambiente intra-hospitalar, estão intimamente relacionados a falha humana (Guilherme et al., 2014).

O paciente vítima de politraumatismo normalmente tem como causador do trauma agentes e situações inerentes a ele e a ambientes hospitalares, como veículos automobilísticos, armas brancas ou de fogo, e agressões físicas por terceiros, o que torna necessário ao paciente um atendimento desde a localidade do seu acontecimento até o âmbito hospitalar, denominação esta, de atendimento pré-hospitalar (Galvão, Rodrigues \& Santana, 2017).

A distribuição dos pacientes de acordo com a etiologia das fraturas é cerca de $36 \%$ das fraturas (39 pacientes) ocorreram em decorrência da violência urbana, como assaltos e agressões, seguidas dos casos de acidentes esportivos (20 pacientes) acidente de trânsito (18 pacientes) e acidentes de trabalhos (7 pacientes). Dos 18 pacientes que sofreram fratura em acidentes de trânsito 13 não usavam cinto ou capacetes. Dos 108 pacientes atendidos 10 estavam alcoolizados e (ou) drogados no momento em que ocorreram as fraturas. A maioria das fraturas ocorreram em pacientes do sexo masculino $(84,04 \%)$, totalizando 89 indivíduos. Foram atendidas 19 mulheres que apresentavam fratura da face (17,6\%). (Bonnati et al., 2005).

$\mathrm{O}$ atendimento pré-hospitalar além de requerer profissionais aptos para desenvolver assistências a saúde do paciente, necessita de transportes devidamente estruturados para realizar o translado do paciente desde a localidade do trauma até o hospital com máxima segurança e agilidade (Mierzwa, Netto \& Oliveira, 2015). Após entrada em âmbito hospitalar, os profissionais devem estar aptos para realizar o atendimento inicial efetivo e lidar com possíveis intercorrências. Foi propagado e instituído como "padrão ouro" pelo American College of Surgeons, o sistema ATLS (Advanced Trauma Life Support) no que diz respeito às vítimas de trauma. Existem ainda outros protocolos de atendimento como o TLSN (Trauma Life Support of Nurses), ACLS (Advanced Cardiology Life Support), PALS (Pediatric Advanced Life Support), a fim de padronizar os 
atendimentos primários e instituir protocolos em seus respectivos setores da área da saúde. Durante a avaliação primária deve ser realizado um exame meticuloso e detalhista da vítima, a fim de impedir riscos iminentes que coloquem a vida do paciente em risco. Para isso, há o sistema $\mathrm{ABCDE}$, onde cada letra corresponde a funções fisiológicas que devem ser avaliadas hierarquicamente e estabilizadas durante o atendimento (Oliveira, 2017).

O ABCDE é um mecanismo que padroniza o atendimento inicial ao politraumatizado e define prioridades na abordagem ao trauma. Entretanto, com aplicação prática dos protocolos, essa sistematização passa por modificações. Esse estudo sistematiza as principais condutas pré-hospitalares e intra-hospitalares que lograram êxito no atendimento ao traumatizado. A letra A (airway) corresponde as vias aéreas e proteção da coluna cervical da vítima. Em vítimas conscientes, deve ser realizada a proteção da coluna cervical com um colar imobilizando a região, entretanto, em pacientes inconscientes, a proteção deve estender-se além da região cervical, englobando todas as outras regiões da coluna, onde tal imobilização deve ser realizada com uma prancha rígida. No que diz respeito às vias aéreas, as mesmas devem ser avaliadas para constatação de possível obstrução com manobras de elevação do mento e anteriorização da mandíbula. Caso seja diagnosticado uma obstrução, existem três procedimentos que podem ser realizados para a desobstrução, sendo eles: intubação endotraqueal, cricotireoidostomia ou traqueostomia. A letra B (breathing) diz respeito a ventilação, onde deve ser avaliado se a respiração está adequada. As vítimas devem ter a região do tórax avaliada, através da inspeção dos movimentos toráxicos, além de avaliar também frequência respiratória, cianose, desvio de traqúeia e observação da musculatura acessória. A letra C (circulation) avalia a circulação e controle de hemorragia, onde as mesmas são estancadas pela compressão direta no foco. Checagem da frequência do pulso, pressão arterial, sudorese, mudança de coloração da pele, e diminuição da consciência podem indicar perfusão comprometida. A letra D (disability) faz checagem quanto ao nível de consciência e estado das pupilas da vítima. Existem dois mecanismos principais para avaliação do estado neurológico do paciente, sendo eles AVPU e Escala de Coma de Glasgow (ECG). O AVPU significa A - alerta, V - responsivo a voz, P - responsivo à dor e U - irresponsivo. Já a Escala de Coma de Glasgow, baseia-se em três parâmetros: resposta motora (varia de 1 a 6), resposta verbal (varia de 1 a 5) e avaliação ocular (varia de 1 a 4), tais parâmetros são acompanhados por escores, onde a sua soma vai determinar o estado neurológico do paciente. Escores entre 13-15, determinam um estado de normalidade, escores entre 9-12 significam que há um dano moderado, e escores entre 3-8 sugerem que o paciente está em estado neurológico severo. Pacientes condizentes a esse escore, devem ser intubados. A letra E (exposition) realiza a análise da extensão das lesões e o controle do ambiente, como por exemplo, prevenção de uma possível hipotermia, com o acompanhamento da temperatura corpórea. Deve ser avaliado também manchas na pele, sangramento e sinais de trauma (Galvão, Rodrigues \& Santana, 2017).

Como principais auxílios no diagnóstico de lesões advindas do trauma, ainda há recursos como a radiografia simples de tórax, e a radiografia de pélvis, que podem ser de grande utilidade para constatação de pneumotórax hipertensivo ou aberto, hemotórax, fraturas de pelve e acetábulo (Caballero et al., 2014). O trauma faz cerca de 16.000 vítimas por dia, dentre as inúmeras regiões do corpo humano que pode acometer, a região facial está elencada como uma das principais, sendo responsável por metade da quantidade de óbitos. A face é composta por estruturas que anatomicamente apresentam maior projeção anterior corpórea, e juntamente com a cinemática do trauma, a sua região torna-se a mais acometida, trazendo distúrbios fisiológicos, funcionais e anatômicos para o paciente (Almeida et al., 2016).

Avaliando a recorrência de trauma facial, Bayley, Bonnick \& Ogundare (2003), relataram ter observado como lesões mais frequentes as fraturas de mandíbula, seguida por lacerações faciais sendo agressão com objeto contundente a causa da maioria das lesões. De acordo com os autores as lesões maxilofaciais são de causa multifacetadas, variando dependendo da região demográfica. Os autores concluem que é necessária uma educação continuada no trânsito e melhorias de condições sociais para que traumas faciais não sejam considerados, uma doença recorrente. 
Observou-se que a faixa etária mais acometida por fratura do esqueleto fixo da face foi a dos 20 aos 29 anos, em 200 casos $(28 \%)$. Dentre as causas encontradas para as fraturas estudadas, a principal foi a agressão física com 280 casos (39,2\%), seguido dos acidentes de trânsito com 105 casos (14,7\%). Houve 18 casos (2,5\%) em outras regiões do corpo, e que numa proporção de 2,78:1 o sexo masculino apresentava-se como de maior número de indivíduos com fratura do esqueleto fixo da face. As fraturas faciais além de acometerem estruturas nobres responsáveis pela audição, visão, e respiração, podem vir a causar riscos iminentes a vida da vítima. São classificadas as fraturas do complexo maxilofacial em: nasal, alvéolo dentária, mandibular, e do tipo Le Fort (I, II e III), podendo ser ainda de forma isolada ou associada (Kaye et al., 2019).

A classificação Le Fort I indica fratura horizontal entre a maxila e o osso palatino. A Le Fort II tem como característica um aspecto triangular, assemelhando-se a uma pirâmide, englobando a região maxilar e do complexo nasal. A classificação Le Fort III, ou disjunção craniofacial, como o próprio nome sugere, ocasiona a separação dos ossos da face das suturas frontozigomática, frontonasal e frontomaxilar, podendo causar ainda a disjunção entre viscerocrânio e neurocrânio resultante da fratura do arco zigomático (Almeida et al., 2016).

Os traumas faciais podem acarretar aos pacientes adversidades, incluindo complicações que requerem uma intervenção hábil e efetiva. Dentre o leque de complicações que podem ocorrer, há duas que requerem atenção, sendo elas: fraturas de base de crânio e obstrução de vias aéreas. Otorragia, Sinal de Battle, blefarohematoma (Sinal de Guaxinim) e rinoliquorréia, são características da fratura de base de crânio. Devido a possíveis fraturas de ossos que estejam intimamente ligados com a manutenção da função respiratória do paciente, a mesma pode vir a ser obstruída, ocasionando empecilhos no caso da intubação orotraqueal, tendo como alternativa, a traqueostomia (Gomes et al., 2017). Pacientes com fratura do complexo maxilofacial podem apresentar ainda maior risco à hemorragia intracraniana, quando comparados a pacientes sem fraturas do complexo maxilofacial, podendo assim, trazer riscos a vida do paciente (Choonthar et al., 2016; Galvão, Rodrigues \& Santana, 2017).

\section{Discussão}

Os autores das pesquisas realizadas no presente estudo, citam o trauma como a principal causa de óbito na sociedade contemporânea, sendo responsável por metade dos índices de óbitos a nível mundial, atribuindo-lhe 5,8 milhões de óbitos anualmente (Gomes et al., 2017; Guilherme et al., 2014; Oliveira, 2017; Affonso et al., 2017). Segundo estudos realizados, as principais situações causadoras do trauma são: acidentes de carro, violência física, e queda, sequência esta que varia em alguns estudos (Caballero et al., 2014; Gomes et al., 2017). No presente trabalho, 67,8\% das fraturas ocorreram em pacientes do gênero masculino e 32,2\% ocorreram em pacientes do gênero feminino. As faixas etárias mais acometidas compreenderam dos 21 os 30 anos com 28,5\%, dos 11 aos 20 anos com 25,2\% e dos 31 aos 40 anos representando 19.6\% dos pacientes (Almeida et al., 2007; Mierzwa, Netto \& Oliveira, 2015; Bonnati et al., 2005).

O protocolo de atendimento Advanced Trauma Life of Support (ATLS) é considerado o padrão ouro para atendimento de urgências e emergências nos centros hospitalares. O sistema ABCDE é um mecanismo que tem por fim padronizar o atendimento inicial ao politraumatizado, além de esquematizar em setores de prioridades a abordagem ao trauma. Conceito o qual, os autores elucidam a literatura (Galvão, Rodrigues \& Santana, 2017; Oliveira, 2017). Os autores do presente estudo corroboram quanto a importância Escala de Coma de Glasgow (ECG), que também é incorporada ao sistema ABCDE, sendo de importância inquestionável para avaliação neurológica do paciente politraumatizado (Mierzwa, Netto \& Oliveira, 2015; Oliveira, 2017; Galvão, Rodrigues \& Santana, 2017).

Quanto as complicações registradas advindas do trauma, vale ressaltar a diminuição significativa de complicações respiratórias, hemorrágicas e osteomusculares. Complicações estas, que apresentam uma maior probabilidade de evolução, sendo tal diminuição, decorrente de uma efetividade no sistema de atendimento inicial ATLS (Caballero et al., 2014; Affonso 
et al., 2017). Dentre as amplas regiões do corpo afetadas em decorrência do trauma, a face está listada entre as mais acometidas, sendo responsável por 35 a 45\% dos índices de traumas (Guilherme et al., 2014; Almeida et al., 2016; Affonso et al., 2017).

O presente estudo registrou uma marcante diferença de incidência de trauma facial entre homens e mulheres $2.82: 1$. Esses valores são consistentes com a literatura. Achou-se também, uma concentração de trauma de face nas $2^{\circ}$ e $3^{\circ}$ década de vida ainda consistente com a literatura. Quanto ao agente causador do trauma de face, a literatura de estudos em realidade sócio econômica destaca o acidente de trânsito (Bonnati et al., 2005). Importante citar que na literatura norte americana, citam a violência interpessoal como principal agente etiológico (Bayley, Bonnick \& Ogundare, 2003; Almeida et al., 2007; Almeida et al., 2016). Quanto a localização e tipos de fraturas faciais mais comuns, os autores se consolidam, elencando a fratura de mandíbula, e as do tipo Le Fort como as mais frequentes (Affonso et al., 2017; Almeida et al., 2016).

Os traumas envolvendo a região de face podem estar intimamente relacionados a diversas complicações que vão requerer um atendimento emergencial ágil e hábil. Dentre tais possíveis complicações, é possível listar duas principais: fraturas de base de crânio e obstrução das vias aéreas (Casulari \& Flores, 2003; Gomes et al., 2017). O presente trabalho pontua para contundo a necessidade de capacitação da equipe de saúde, para melhor servir ao crescente número de trauma facial que recebem atendimento de emergência em via pública corroborando com os autores (Caballero et al., 2014; Affonso et al., 2017).

Segundo Gomes (2017) o primeiro atendimento ocorre a utilização do protocolo universal ATLS, mas posteriormente o atendimento torna letificado pois a equipe fica sem autonomia e direcionamento quanto a continuidade do processo desse atendimento. Um treinamento deve ser desenvolvido para oferecer condições seguras para uma atuação efetiva da equipe em situação de emergência, com o propósito de estabelecer precocemente interrupção no ciclo de desequilíbrio funcionais que levam a morte. Envolvendo assim, um preparo para avaliar as condições do paciente e um profundo conhecimento do equipamento da área que recebe o paciente. Além disso, o uso de equipamento (aspirador, ventilador e desfibrilador) e o papel a ser desempenhado na equipe (Bayley, Bonnick \& Ogundare, 2003; Almeida et al., 2007; Almeida et al., 2016).

\section{Conclusão}

Pode-se concluir que as lesões na região da face são causadas geralmente, devido a trauma e acidentes incidindo na grande maioria das vezes na população jovem. A incidência pode ser reduzida por meio de medidas educativas visando a diminuição do consumo de álcool e a segurança do trânsito como uso de capacete e cinto de segurança. Além disso, observouse que frequentemente os traumas estão associados a complicações envolvendo base de crânio e emergências neuro cirúrgicas. Nesse contexto, ressalta-se que o atendimento e tratamento precoce é fundamental para uma técnica bem-sucedida, além de uma conduta multidisciplinar e integral.

\section{Referências}

Affonso, P. R. A., Cavalcanti, M. A., Gandelman, I., \& Groisman, S. (2017). Etiologia de Trauma e Lesões Faciais no Atendimento Pré-Hospitalar no Rio de Janeiro. Revista UNINGÁ. 1(23):10.

Almeida, D. B., Beccaria, L. M., Matta, P. R. A., Poletti, N. A. A., Soller, I. C. S., \& Squizatto, R. H. (2016). Epidemiological Profile of Patients with Facial Injures Treated in Emergency Hospital. Rev. Min. Enferm. 20(1):935.

Almeida, P. F., Camargo, L. M., Macedo, J. L. S., \& Rosa, S. C. (2007). Mudança Etiológica do Trauma de Face de Pacientes Atendidos no Pronto Socorro de Cirurgia Plástica do Distrito Federal. Rev. Bras. Cir. Plás. 4(22):10.

Bayley, N., Bonnick, A., \& Ogundare, B. O. (2003). Pattern of Mandibular Fractures in na Urban Major Trauma Center. Journal of Oral na Maxillofacial Surgery. 6(61): 713-718.

Bonnati, B. D., Borba, B. H. C., Costa, J. M. C., Patrocínio, J. A., Patrocínio, L. G., Pinto, L. F., \& Vieira, J. L. (2005). Fratura de Mandíbula: Análise de 293 Pacientes Tratados no Hospital de Clínicas da Universidade Federal de Uberlândia. Rev. Bras. Otorrinoralingo. 5(7):10. 
Research, Society and Development, v. 10, n. 1, e7210111530, 2021

(CC BY 4.0) | ISSN 2525-3409 | DOI: http://dx.doi.org/10.33448/rsd-v10i1.11530

Botter, D. A., Parreira, J. G., \& Wulkan, M. (2005). Epidemiology of Facial Trauma. Rev. Med. 5(51):10.

Caballero, F., Cáceres, E., Koo, M., Monerri, M. M., Montmany, S., Muñoz-Vives, J. M., Navarro, S., Orrego, C., Pobo-Perris, A., Prat, S., \& Puig, S. (2014).

Estudio Para la Mejoría de la Atención Hospitalaria Inicial del Paciente Politraumatizado: Proyeto Traumacat. Med. Clin. 1(143): 1-80.

Campos, A. L. (2015). Atendimento de Emergência Realizado por Profissionais de Enfermagem, Médico, Bombeiros e Demais Profissionais Treinados a Vítimas de Acidentes e Catástrofes. Rev. Med. 1(4):10.

Casulari, L. A., \& Flores, D. S. (2003). Blefaro-hematoma, Otorragia e Sinal de Batle como Indicadores de Fratura de Base do Crânio e de Lesões Intracranianas. Brasília Médica. 1(40): 43-45.

Choonthar, M. M., Pandaya, K., Pradeep, S., Prasad, R., \& Raghothaman, A. (2016). Head Injury - A Maxillofacial Surgeon's Perspective. Journal of Clinical \& Diagnostic Research. 1(10):10.

Galvão, I. M., Rodrigues, M. S., \& Santana, L. F. (2017). Utilização do ABCDE no Atendimento do Traumatizado. Rev. Med. 4(96):10.

Gomes, R. S. S., Luz, F. A., Machado, A. N., Mattos, L. L., Sales, E. C., \& Santiago, L. G. (2017). Pacientes Acometidos por Trauma Grave de Face: Abordagem, Etiologia, Prognóstico e Características. Anais do Seminário Científico da FACIG. 3(1):10.

Guilherme, F. J. A., Jesus, R. F., Silva, R. C., Santos, L. N. C., \& Santos, M. B. (2014). Avaliação Primária no Atendimento ao Trauma em Ambiente de Simulação. Almanaque multidisciplinar de pesquisa. 1(2):10.

Kaye, R., Paskhover, B., Povolotskiy, R., \& Youssef, P. (2019). Facial Fractures in Young Adults: A National Retrospective Study. SAGE Journals: Annals of Otology, Rhinology and Laringology. 1(1):10.

Mierzwa, T., Netto, F. S., Oliveira, A. (2015). Protocolo de atendimento inicial ao politrauma HUOP: princípios práticos de organização de saúde. SAGE Journals: Annals of Otology, Rhinology and Laringology. 1(1):10.

Oliveira, T. N. S. (2017). Trauma: Atendimento Inicial no Intra-Hospitalar. Repositório Instucional da UFSC. 1(1):10. 